\title{
The significance of coagulative and thrombotic changes after haemorrhage and injury
}

\author{
R. M. HARDAWAY \\ Washington, DC, USA
}

Haemorrhagic shock may progress under certain circumstances by the events described in the diagram shown in Figure 1. Normally, arterial blood pressure is adequate: arterioles are open, though with adequate tone; arteriovenous shunts are closed; and only about $20 \%$ of the capillaries are open at any one time. Flow is so good in the functioning capillaries that the cells are satisfied with about $20 \%$ perfusion time. When mast cells lining and adjacent to a closed capillary become slightly anoxic, they secrete histamine which causes the capillary to open. The histamine is destroyed in the blood quickly and does not circulate. When the mast cells are satisfied, they halt histamine secretion and the capillary closes. In this way the capillaries take turns in being perfused. Capillary flow is rapid and the fall in $p \mathrm{H}$ along the capillary issmall, perhaps from $7 \cdot 4$ to $7 \cdot 3$. Lactic acid is continuously being excreted in minimal amounts by the cells and carried away by the capillary flow; normally this is so good that lactic acid does not accumulate. The sequence of events in haemorrhagic shock is as follows.

\section{Phase I: Reversible Haemorrhagic Shock}

\section{STAGE 1: VASOCONSTRICTION}

Initiated by some shock-producing stress such as a haemorrhage or trauma, the sudden secretion of catecholamines and the high blood levels of these emergency hormones initiate an intense arteriolar vasoconstriction. This stage is that of compensated or partially compensated shock and blood pressure may be normal. However, the pulse may be rapid and thready. There is other evidence of sympathetic stimulation: pale, sweaty skin, rapid, thready pulse, elevation in blood glucose, and general nervousness. These are common clinical findings associated with shock. The çatecholamin level in the blood remains high throughout shoc even until death.

STAGE 2: CAPILLARY AND VENULE OPENIN This might also be called the stage of expansion of the vascular space by the opening of capacitance vessels. It is also the stage of hypercoagulable and stagnant capillary blood. Acidosis is a prominerif feature of this stage. It is easily reversible bo volume administration. As seen in Fig. 1, arter? olar vasoconstriction with or without a fall is arterial blood pressure results in less volume of blood flowing through the arteriole. Therefore capillary flow slows. Parenchymal cells, which are usually satisfied with intermittent perfusion of their particular capillary, now find they need longer and longer perfusion times to maintain $\overline{3}$. normal metabolism. Mast cells adjacent to each capillary provide for this by secreting sma丑 amounts of histamine when they become slightly anoxic. This causes the opening of that particular capillary, and keeps it open as long as the histamine is secreted. The histamine is quick $\overline{\bar{V}}$ destroyed in the bloodstream so that other vessers are not affected. However, as each capillary andes venule remains open longer in this way, aft increasing proportion of capillaries are open any one time until, when carried to extreme, the are nearly all open at once. This particular applies to the abdominal viscera. Because of the tremendous number of capillaries and venules it the body, a large part of the blood volume $\overline{\mathbf{s}}$ always in these vessels (capacitance vessels), ev under normal conditions with only about $20 \%$ off capillaries open at one time. With the opening of many additional capillaries and venules the total volume of the vascular space may actually double This is reflected in extreme congestion of viscers such as thelung, liver, kidneys, and gastrointestinc需 
mucosa. Those who rely on measurements of blood loss to gauge volume replacement are often fooled by this phenomenon. A frequent comment is that more blood was lost than is realized. This capillary and venule expansion has two important effects on the shock picture. First, the vascular space is greatly expanded. This makes even a normal blood volume inadequate to fill it. The capillary and venule capacity is so great that very little blood is left over to reach the great veins and venae cavae. Vena caval pressure falls to zero, resulting in a great reduction of venous return to the right atrium. Then the cardiac output falls still further, and also arterial pressure. Second, the low blood pressure, extremely constricted arterioles, open arteriovenous shunts, and many open capillaries together produce an extremely stagnant capillary flow. This causes slow delivery of oxygen and increased anoxic metabolism. With increased secretion of lactic acid into the slow-moving capillary blood, the blood becomes acidic by the time it reaches the venous end of the capillary. A marked lactic-acidaemia develops quickly, first in the venous blood and later also in the arterial blood. The body compensates for the acidaemia by blowing off $\mathrm{CO}_{2}$ till the excess lactate is eventually removed. In fact, in early shock this

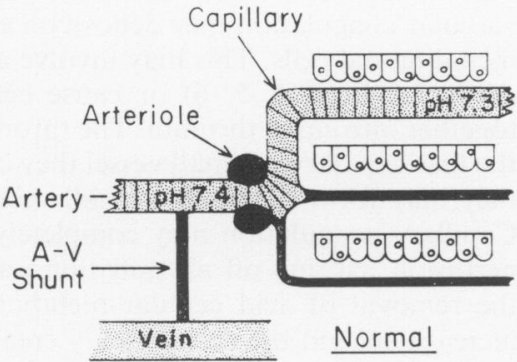

2.
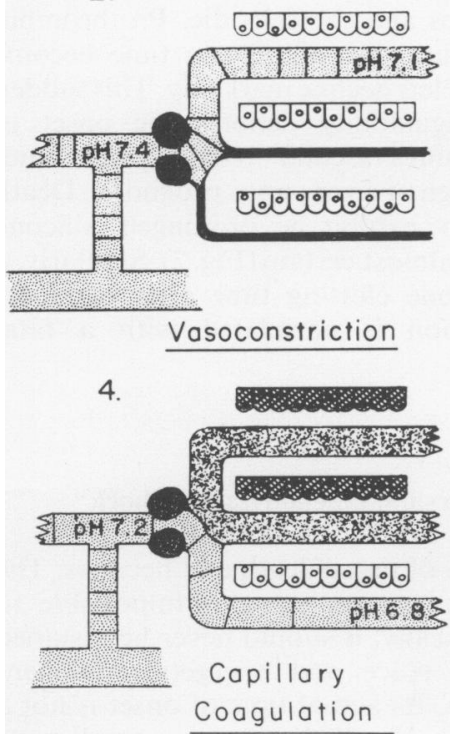

3.
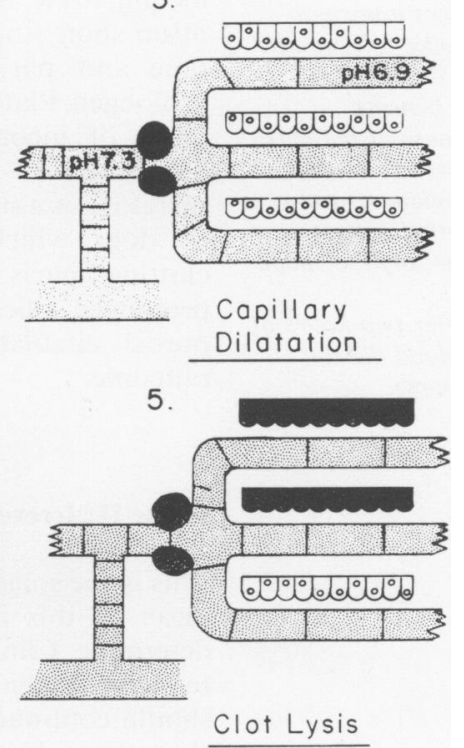

compensatory mechanism may even overcompensate and produce a respiratory alkalosis. However, compensation becomes inadequate as the lactic acid level continues to increase and the $p \mathrm{H}$ falls, producing a true acidosis. This acidosis has a number of effects, including a marked effect on blood coagulability. Its full extent will not be realized if one merely takes the $p H$ of arterial blood, because it is in the veins where it is most prominent. In addition, certain vascular beds will be much more affected, that is, where vasoconstriction is worst as in the abdominal viscera. Blood $p \mathrm{H}$ levels as low as 6.6 have been recorded here. Further, arteriovenous shunting may help to raise the venous $p H$. This may give a false sense of security. Capillary and tissue $p \mathrm{H}$ is probably much lower. Very important is the tremendous increase in lactic acid which must occur around cells which are denied perfusion. Anaerobicmetabolism, which soon comes on, increases this. Delicate cellular enzymes cannot work under these conditions.

The effect of this acidosis on blood coagulation is dramatic. It produces a hypercoagulable state. The silicone clotting time may fall from a normal of 20 to 40 minutes to three to five minutes (Figs. 2, 3 ). The sluggish hypercoagulable blood may not

Fig. 1 Stages in Development of Irreversible Shock Diagram represents a small artery ending in an arteriole with sphincter-like action. Arteriole feeds three capillaries, each feeding a group of cells.

1 Normal Condition Arterioles fairly widely opened. Only one capillary is being perfused while others rest. Capillaries open in rotation on demand $o_{s}$ the cells adjacent to them. Constant perfusion not necessary. Blood flow through the capillary is rapid. $\mathrm{p} H$ drop across the capillary is minimal.

2 Phase I: Shock Vasoconstriction Due to catecholamine secretion after haemorrhage and trauma. Arteriole constricts letting less blood through. Hypotension in the artery, capillary flow slowed.

3 Phase II: Shock Capillary Dilatation (Expansion of Vascular Space) All capillaries are open on demand of their cells. Blood flow through all capillaries is extremely slow. $\mathrm{pH}$ fall across the capillary is marked.

4 Phase III: Shock-disseminated Intravascular Coagulation Late shock. Haemolysis has aided the slow flowing acid blood in two of the capillaries to clot, stopping perfusion completely in these capillaries. Cells nourished by these capillaries are dying. Blood flow through the remaining capillary is sluggish. Circulating blood incoagulable.

5 Phase IV: Irreversible Shock Arteriole has opened somewhat after replacement of blood volume. Capillary clots have been lysed by endogenous fibrinolysin thereby restoring circulation. However, cells supplied by the formerly clotting capillaries are now dead. This produces areas of focal necrosis and if widespread enough, causes tissue and organ failure. 
clot in vivo and the shock may not progress. Pure haemorrhagic shock is almost always reversible up to the point of death. Of course if the mean arterial pressure drops below a critical point $(20 \mathrm{~mm} \mathrm{Hg})$, capillary perfusion halts and death ensues. Up to this point, pure haemorrhagic shock responds well to adequate treatment.

However, many factors are possibly present which can initiate in vivo coagulation in this

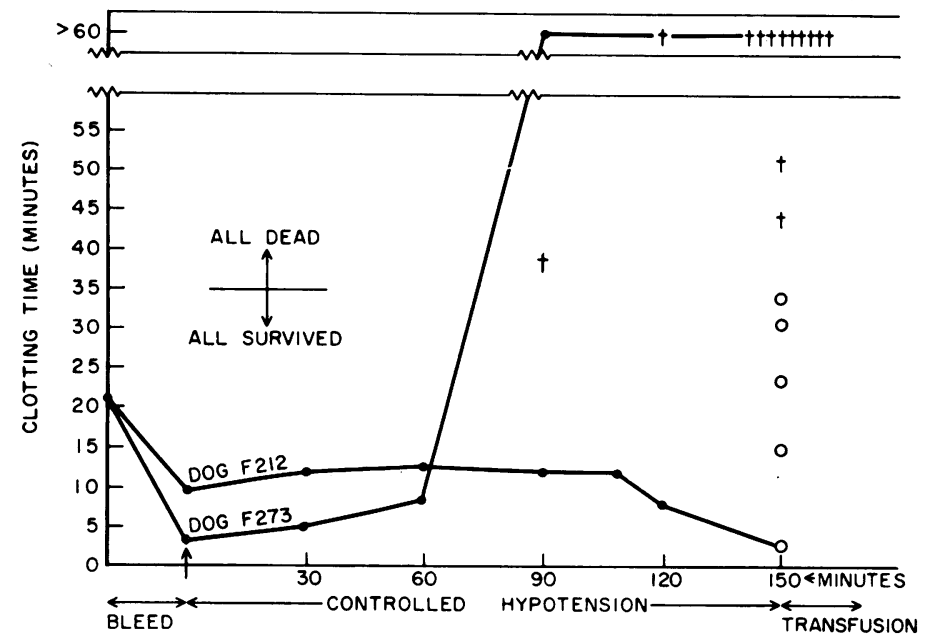

Fig. 2 Silicone clotting times of dogs subjected to haemorrhagic shock. Bleeding produced a decrease in clotting time. The dogs which later developed a prolonged clotting time (over 35 minutes) all died as noted by the crosses. Those which did not all survived, as noted by the circles. Two typical animals are shown by the lines.

Fig 3. Thromboelastograms of the blood of a dog subjected to haemorrhagic shock. The record at the top was taken before haemorrhage and shows a normal clotting configuration consisting of a spike followed by a torpedo shape. The spike or straight line portion denotes fluid blood. The torpedo shape beneath denotes rapidity of formation and firmness of the clot.

The second recording (just below) was taken after haemorrhage and shows an almost complete absence of the spike showing that the blood clotted almost immediately. The increased width of the torpedo shape denotes rapid and firm clotting.

The third recording (below) taken after two hours of shock shows only a straight line. The blood did not clot during the recording (about two hours). dangerously susceptible blood. If the oligaemia not corrected by adding to the volume (more thă a normal blood volume is required) and if it aided by even a mild amount of trauma, infection, haemolysis, or other clot-initiating factors, the shock may enter the next stage, that of dissennated intravascular coagulation.

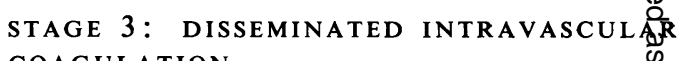
COAGULATION

This stage is roughly equivalent to 'refractor $\vec{y}$ ', shock, that is, shock which is resistant to treatment but is still reversible. Many capillarfs become occluded by clotted blood (Fig. 1). Thte stage is ushered in by the sudden appearance $8.8 \mathrm{f}$ incoagulable or hypocoagulable blood. Clinicalfy, widespread oozing of blood from wounds mây suddenly appear. Previously adequate haemiestasis seems to become inadequate. It may be impossible to stop the bleeding by ligatuge, pressure, or other means. The coagulation within capillaries has two important consequences. Firgt, the widespread coagulation of large quantities blood in the expanded capillary bed uses up lange quantities of clotting factors, thereby leaving the remaining unclotted blood deficient in clottipg factors and hence hypocoagulable or incoagulabfe. Second, fibrin formed by disseminated int荋vascular coagulation may deposit on any surfaee, including red cells. This may involve a thick corat of fibrin (Figs. 4, 5, 6) or cause cells to st together into small thrombi. The thrombi occlu the first capillary or small vessel they come to. $\mathrm{Gr}$ they may form in situ in a small vessel (Fig. $\$$ ). Capillary coagulation may completely halt local perfusion, cutting off all nutrition and stopping the removal of acid cellular metabolites. These increase around the cells quickly creating suclpa marked local acidosis that the $p \mathrm{H}$ falls to levels incompatible with cell enzyme activity; metahbolism soon stops and the cells die. Prothrombin time and partial thromboplastin time become prolonged. Platelets decline markedly. This sudfon onset of incoagulability denotes the onset fof disseminated intravascular coagulation anf, therefore, is a sign of a very poor prognosis. Deafh . of dogs which exhibit a prolonged siliconge clotting time is almost certain (Fig. 2). Similarlypa prolonged silicone clotting time after extrac $\hat{\beta}^{*}-$ poreal circulation is correlated with a fatal outcome.

\section{Phase II: Irreversible Haemorrhagic Shock}

This is the stage of tissue death and necrosis. The onset of this important stąe is impossible To determine. Clinically, it should never be assumed to have taken place and energetic treatment should continue. Its actual time of onset is not a sharp line; death of cells begins on a small sca्युe 


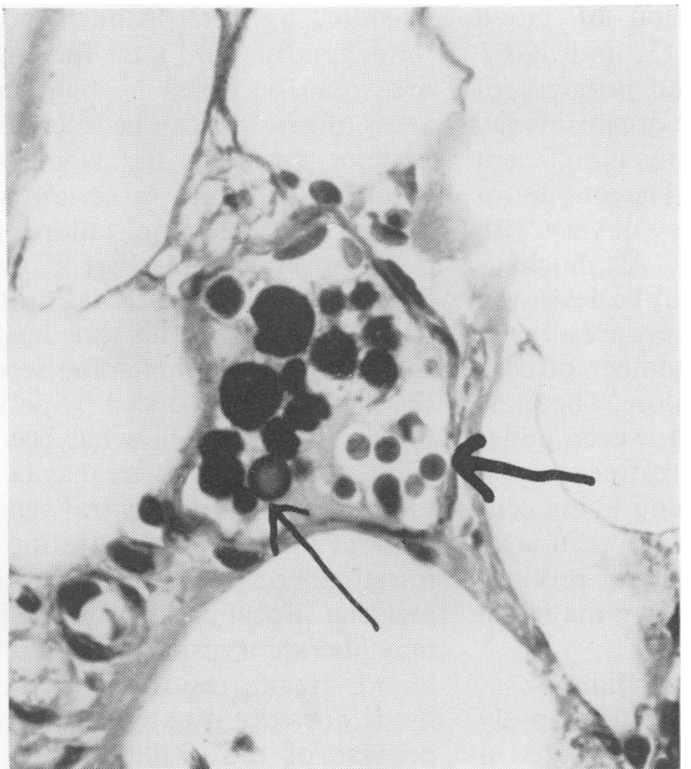

Fig. 4 Small vessel in a patient dying of multiple trauma. Section is stained by PTAH and aniline-eosin which renders fibrin blue-black, while allowing red cells to remain red. A normal red cell is seen in the centre, and above it (right hand arrow) another which has taken on an additional bluish hue. The other arrow shows a larger globule with a haemoglobin centre and a blue-black coating. At the top are a cluster of heavily staining globular clots. $\times 700$. Fibrin formed in the flowing blood may deposit on any surface including the surface of red cells.

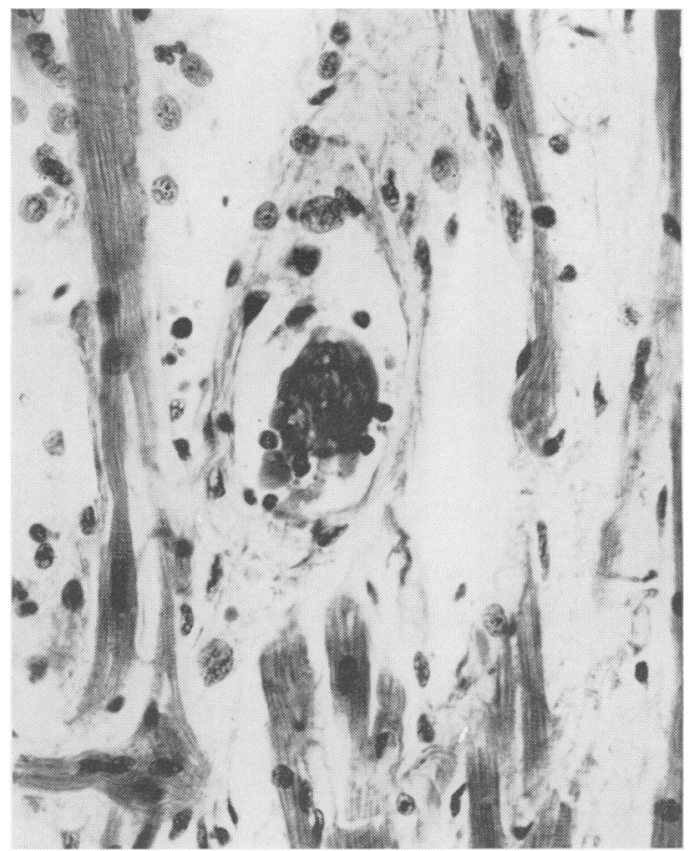

Fig. 6 A microthrombus in a small myocardial vessel of a 60-year-old woman dying from haemorrhagic shock. Note the surrounding oedema but with normally preserved myocardial fibres. $H \& E, \times 350$.

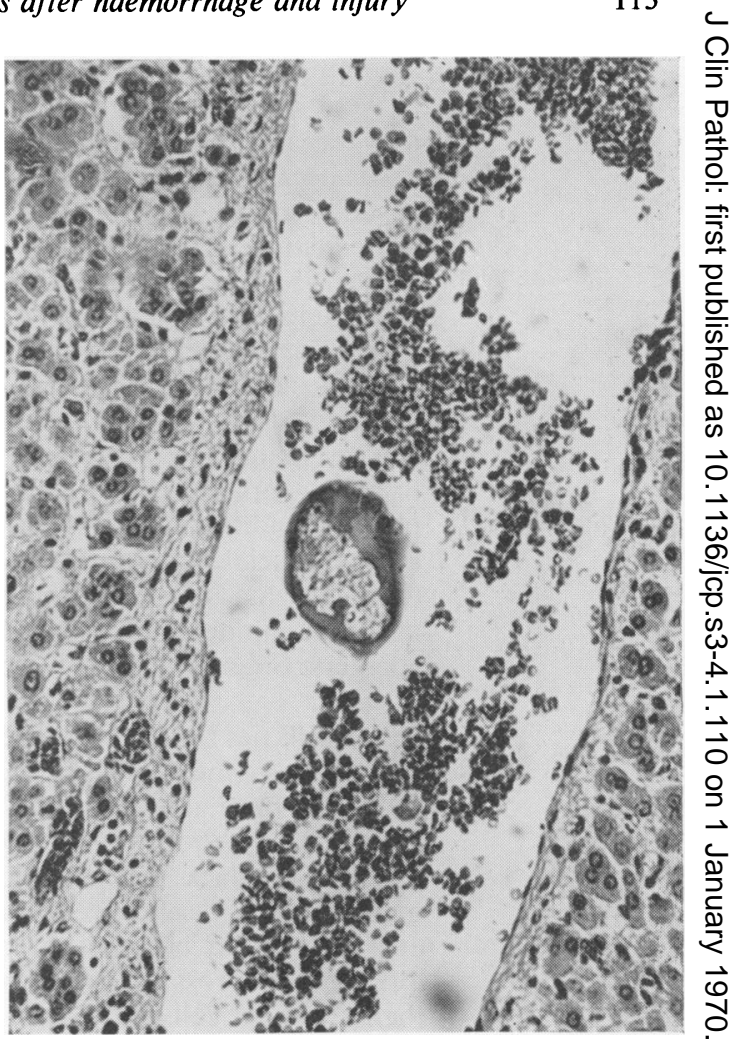

Fig. 5 Central vein of the adrenal gland containing a globular clot. The amorphous, poorly staining centre and three trapped red cells are seen. $H \& E, \times 360$.

From a 50-year-old man who died after acute pancreatitis with renal failure and profound shock.

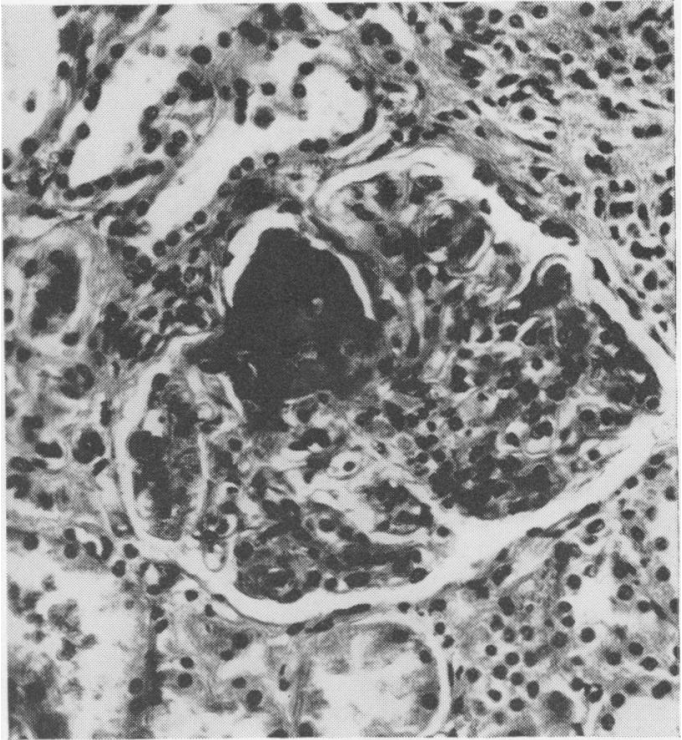

Fig. 7 Fresh microthrombus in a glomerulus of a 75-year-old man dying from septic shock secondary to Pseudomonas pneumonia. $H \& E, \times 192$.

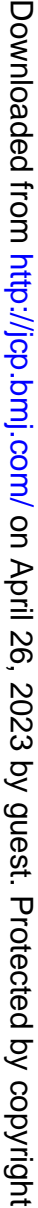


and progresses. The determination of blood enzyme levels such as LDH, SGOT, and SGPT may be an index of the onset and progress of tissue necrosis. Death of the whole organism may ensue when cell death in vital organs is sufficient to be incompatible with function. The amount of necrosis required for organ failure will vary with the original health of the organ. As much as three-quarters of normal kidneys may be destroyed with adequate function; but if kidneys are already diseased, death of only a small number of cells will be enough to produce renal failure. The same principle applies to other organs. However, under proper conditions of supportive treatment (such as an artificial kidney) the remaining living cells (kidney or liver) may multiply and reconstitute an adequate amount of parenchyma to produce adequate organ function; the organism may then survive.

If capillaries remain occluded for an hour or so, the cells nourished undergo changes which rapidly become irreversible. Lack of oxygen results in anaerobic metabolism with increased lactic-acid production; lack of perfusion increases acid metabolites, and cellular enzymes cannot function at a low $p \mathrm{H}$. It becomes impossible to maintain differentials across the cell membrane: potassium then leaves the cell and sodium enters it. The cells become unable to metabolize raw materials and metabolism stops. Soon, certain changes can

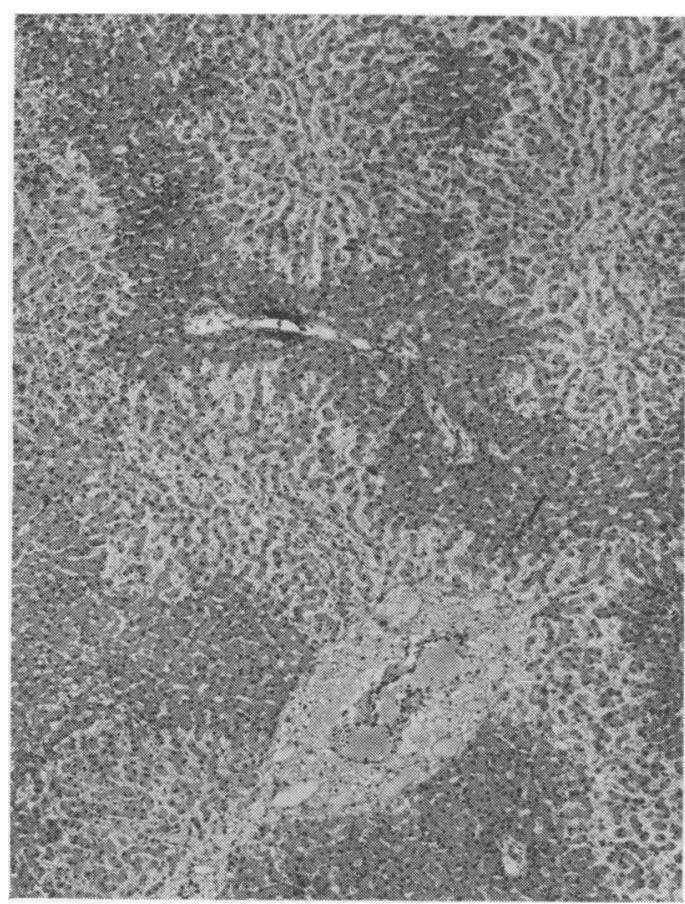

Fig. 8 Liver of a dog dying of endotoxin shock. Note marked congestion and centrilobular necrosis. $H \& E$, $\times 110$. be noted by electron microscopy, first affecti⿳亠二口犬g mitochondria, and later focal necrosis in certain areas can be noted by light microscopy. Large areas of necrosis can be tolerated in many orgahs without death of the whole organism, but $\exists$ enough tissue dies in certain vital organs, that organ fails. Hepatic failure is perhaps mast common and the earliest in onset (Fig. 8). It heralded by a dramatic fall in the blood glucose level, which up to this time has been high due 甲o the effect of catecholamine secretion. Failure 腃 the kidneys is evidenced by persistent anuria; 由 to this time, urine flow has been reduced or onily transiently halted. There may be evidence of heaut failure with a rising central venous pressure even without volume administration (Fig. 9). Gastrôintestinal necrosis may be denoted by the onset \&ff melaena. Focal areas of necrosis in the pancreas may liberate trypsin which, if it gainsaccess to the blood stream, results in further clotting. Lung tissue does not usually become necrotic, perhaps because of the availability of oxygen and the relatively low metabolic requirements of its connective tissue and endothelium. Howevê,, thrombosis may produce a severe picture haemorrhage into alveoli leading to death from respiratory failure. The picture is that of haemorrhagic infarction. Death of the organism may follow from any or all of these organ failures.

A review of the cases of the 'crush syndrome'

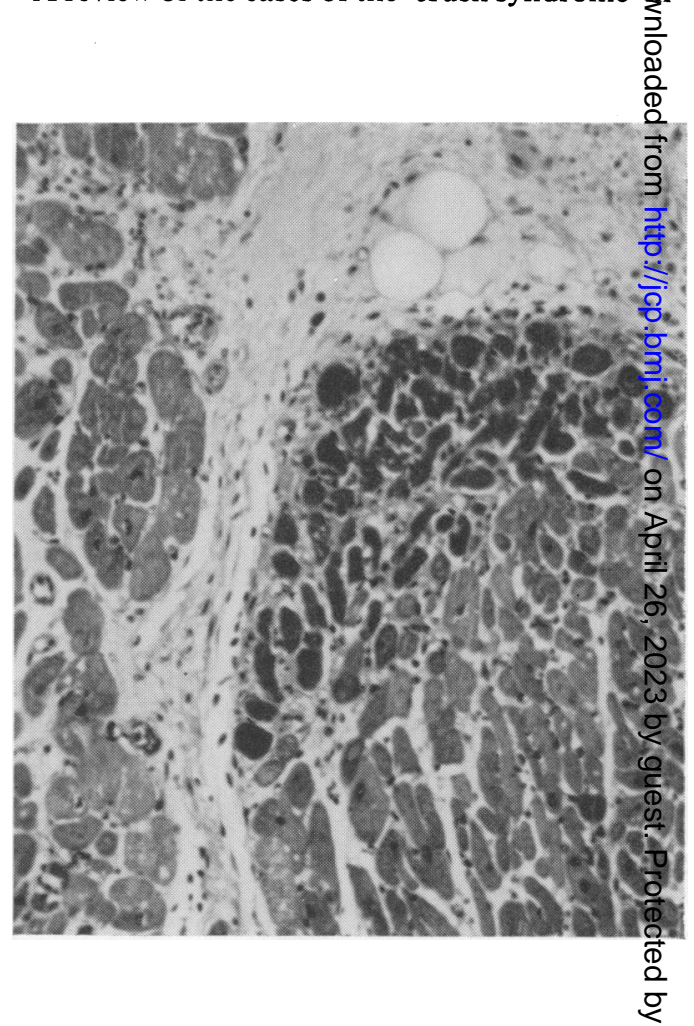

Fig. 9 Microinfarct of the myocardium. Patient die of Gram-negative septicaemia. There were many globular clots in various tissues, including the heart. $H \& E, \times 110$. 

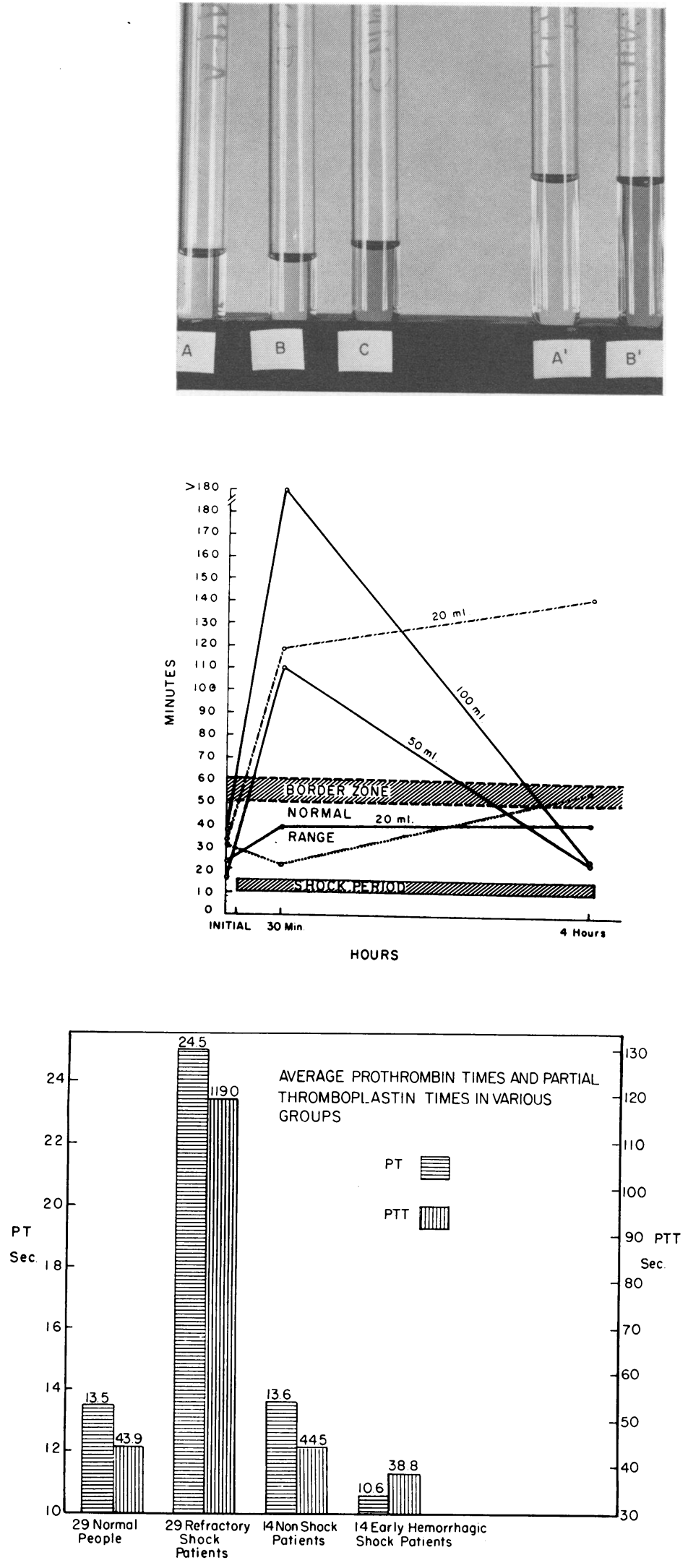

Fig. 10 On the left are three blood samples taken from a dog subjected to 100 blows of a padded mallet on one thigh. Blood in tube A was drawn before the trauma, in tube B 30 minutes later, and in tube C 48 hours after the trauma. Note that haemolysis, which was only slight soon after the trauma, had increased markedly by the end of two days. This is thought to be due to absorption of haematoma and ecchymosis probably via lymphatics. If no haemolysed blood had been absorbed, the mild haemolysis shown in tube B would have disappeared completely in a few hours. On the right are two tubes of blood drawn before $\left(\mathrm{A}^{1}\right)$ and after $\left(\mathrm{B}^{1}\right)$ the administration of $20 \mathrm{ml}$ of autogenous blood which had been frozen and thawed. Note that the amount of haemolysis 48 hours after trauma and immediately after the administration of $20 \mathrm{ml}$ of haemolysed blood is about the same.

Fig. 11 Silicone clotting times in groups of dogs. Dogs subjected to haemorrhagic shock alone show a decrease in silicone clotting time with a subsequent lengthening at the end of the shock period into a borderline zone of 55 minutes. Three groups of dogs given haemolysed blood without any shock procedure, show varying changes according to dosage of haemolysed blood but all are within the normal range by four hours. Dogs given 50 and $100 \mathrm{ml}$ of haemolysed blood showed a transient but dramatic prolongation of silicone-clotting times at 30 minutes after administration of the lysed blood. In dogs given $100 \mathrm{ml}$, the 30-minute samples often did not clot in a week. Dogs subjected to a haemorrhagic shock after administration of $20 \mathrm{ml}$ of haemolysed blood showed an immediate lengthening of clotting time characteristic in the dogs given 50 or $100 \mathrm{ml}$ of haemolysed blood, but instead of returning to normal levels within four hours, there was a further increase in clotting time. This prolonged clotting time at the end of a shock period is characteristic of irreversibility. Thus, the initial shortening of clotting time after haemorrhage is converted to a marked prolongation by prior administration of $20 \mathrm{ml}$ of lysed blood $\underline{-}=$ haemolysed blood alone; $\cdot-\cdot-\cdot=20 \mathrm{ml}$ haemolysed blood and shock -.. = shock alone.

Fig. 12 Average prothrombin and partial thromboplastin times in various groups of patients and normal people. In unshocked patients the prothrombin times and partial thromboplastin times are normal. In early haemorrhagic shock these tend to be shortened. This may indicate a hypercoagulability which may predispose toward disseminated intravascular coagulation and the development of a clotting defect. In refractory shock there is marked prolongation of prothrombin time and partial thromboplastin time indicating a clotting defect. 
the literature reveals several with a haemorrhagic diathesis. Bywaters (1942) noted that haemorrhage could be either internal or external and was the most common complication of this syndrome. The mechanism of this haemorrhagic diathesis was not then known. Because of the similarity of this syndrome to the incompatible transfusion syndrome, the possibility arose that the haemorrhagic phenomena in both might be due to the same mechanism.

There was a clinical impression that patients with soft-tissue injury did not tolerate haemorrhage as well as uninjured subjects. Simple haemorrhage was tolerated quite well by dogs and caused neither death nor evidence of disseminated intravascular coagulation as indicated by the lack of change in the clotting mechanism. Therefore, it was thought that dogs with injury to one limb caused by multiple blows to the thigh (under anaesthesia) with a rubber hammer might be more susceptible to haemorrhagic shock, due perhaps to the liberation of tissue thromboplastin into the blood stream. This was tried but proved not to be the case. The animals were not apt to die even though they incurred both trauma and a degree of haemorrhagic shock which was not fatal. This was unexpected and some explanation was sought. Then the work of Borgström, Gelm, and Zederfeldt (1959) came to our attention and seemed most pertinent. They ligated both femoral veins of rabbits and then administered blows with a mallet to one thigh; thrombi formed in the femoral veins of both the injured and uninjured limbs, the incidence reaching a peak by 48 hours after injury. This indicated stimulation of intravascular coagulation by a factor or process present at peak levels at about 48 hours. Our doz experiments were then repeated allowing a 48-hour interval between trauma and haemorrhage: now the results were different, death usually resulting from haemorrhage which otherwise was not fatal. In studying blood samples of the dogs, it was noted that though there was little or no haemolysis one half hour after trauma, a marked haemoglobinaemia developed by 48 hours (Fig. 10). One hundred blows to one thigh resulted in a moderately swollen and discoloured thigh which was not at all disabling two days later. It was not certain whether the pigment was haemoglobin or myoglobin, but it was surprising that its excretion increased over a two-day period in the face of normal renal output. The effects of an equivalent amount of haemolysis without any trauma were then investigated. The haemoglobinaemia produced after 48 hours could be roughly duplicated by removing $20 \mathrm{ml}$ of the animal's blood, freezing it, thawing it, and returning it to the animal. This amount of haemolysis had no demonstrable effect alone. In fact, the administration of $100 \mathrm{ml}$ of autogenous haemolysed blood was also without obvious effect. However, other experiments proved that even the $20 \mathrm{ml}$ dose was capable of producing dramatic effects. The following observations were made on dogs suffering from haemorrhagic shock (Fig. 11).

1 A small amount $(20 \mathrm{ml})$ of haemolysed blood, given before haemorrhagic shock is produced, causes an increase in mortality of dogs from $13 \%$ to $91 \%$.

2 This lethal effect is due to the stimulation by흠 haemolysed blood of intravascular coagulation. $\frac{\bar{s}}{\bar{a}}$ However, in normal rapid capillary flow, coagu- $\stackrel{\mathbb{\Omega}}{\Omega}$ lation does not take place. A second factor is necessary, namely, stagnant capillary flow as produced by arterial hypotension, arteriolar ${ }^{\circ}$ vasoconstriction, and capillary dilatation. All of $\vec{\omega}$ these are produced by an otherwise non-lethals haemorrhage.

3 The lethal factor of haemolysed blood is ais clotting factor (thromboplastin) in red cells.

4 Large amounts $(100 \mathrm{ml})$ of haemolysed blood alone are harmless, but even a small amount $\overrightarrow{-}$ $(20 \mathrm{ml})$ is lethal in the presence of shock.

5 Haemolysed blood causes the conversion of fibrinogen to fibrin.

6 Therapeutic 'fibrinolysin' will prevent death in otherwise irreversible haemorrhagic shock either produced by haemorrhage alone or when influenced by haemolysed blood.

7 Endogenous heparin secretion is stimulated byo disseminated intravascular coagulation produced either by haemorrhage alone, haemolysed blood alone, or by a combination. It is probably protective mechanism.

8 A markedly prolonged silicone clotting timê occurring during haemorrhagic shock is an accurate prognostic index of irreversibility.

9 Irreversibility is correlated with a fall in the plasma fibrinogen level when that is due to consumption in disseminated intravasculak coagulation.

Red cells contain a thromboplastin whiclo initiates blood coagulation. How ever, fairly large scale haemolysis is harmless in a normal circula tion; intravenous injection of distilled water is harmless to normal humans, and the only effect? are transient haemoglobinaemia and haemoglobin uria. Injection of $100 \mathrm{ml}$ of autogenous haemolyse blood into normal animals produced no harmfū results but it did produce a transient, but? dramatic, prolongation in silicone clotting time, $\widetilde{\infty}$ transient rise in prothrombin time, a transient rise in endogenous heparin, and a mild an $\$$ transient fall in the fibrinogen level. All returned to normal within four hours. However, whe combined with otherwise non-fatal haemorrhagic shock, even 20 ml of autogenous haemolysed bloo was fatal in over $90 \%$ of animals. It also produce a significant increase in fibrinogen utilization, an 8 increased clotting and prothrombin times. The effects were progressive over the four-hour shock period. The fatal effect of haemolysed blood 8 attributed to initiation of an episode of dissemp nated intravascular coagulation. It was not pres. duced by an injection of pure haemoglobin. It postulated that the shock-induced stagnant 
capillary circulation, plus the initiation of coagulation by haemolysis, caused clotting of the blood in the microcirculation, and that haemolysis initiates coagulation by the liberation of red-cell thromboplastin. That the toxic effects of haemolysed blood are due to its stimulation of disseminated intravascular coagulation is supported by the protective effect of fibrinolysin. Fibrinolysin administered during haemorrhagic shock after the administration of $20 \mathrm{ml}$ of autogenous haemolysed blood reduced the mortality from 91 to $38 \%$. Fibrinolysin acts by lysing capillary thrombi.

\section{Clinical Studies}

Twenty-nine patients in refractory shock were studied at the Walter Reed shock unit. All developed a coagulation defect (Figs. 12-15). Table $I$ is a summary of the results. It was not possible to associate clearly a coagulation abnormality with the shock syndrome in every case, because of preexisting diseases and/or therapeutic agents administered before the onset of shock.

In 22 patients, the coagulation abnormality was definitely associated with the shock syndrome and no other aetiology was possible. Five had severe infection, four giving positive blood cultures. Bacteraemia was suspected in the other but blood culture was negative probably because of antibiotic therapy.

Coagulation studies were carried out on 130 cases of shock at the Third Surgical Hospital in Vietnam. The general character of these patients was different from those at Walter Reed. The Vietnam cases generally had severe oligaemic shock from battle wounds. However, most were easily restored to a normal condition by transfusion and only a few could be regarded as 'refractory' shock, unlike nearly all the cases studied at the Walter Reed Hospital.

Sixty of the 130 cases had coagulation abnormalities. In general, these were of two types, hypercoagulability and hypocoagulability. Evidence of hypercoagulability was found in 14 subjects with prothrombin times less than 11 seconds or partial thromboplastin times below 30 seconds (Fig. 12). This may be analogous to the hypercoagulable phase of haemorrhagic shock in dogs discussed earlier (Fig. 2). Evidence of hypocoagulability was found in 46 patients who had prolonged prothrombin times (over $16 \mathrm{sec}$ ) or partial thromboplastin times (over $75 \mathrm{sec}$ ). These, in general, were the most severely shocked patients. Increasing severity of clotting defects was associated with death whilst a rapid spontaneous repair of the clotting defect was associated with recovery.

Another group of 45 seriously wounded patients were studied at the 93rd Evacuation Hospital in

\begin{tabular}{|c|c|c|c|c|c|c|c|c|c|c|c|c|c|}
\hline \multirow[t]{2}{*}{ Patient } & \multirow{2}{*}{$\begin{array}{l}\text { Plasma } \\
\text { Fibrinogen } \\
(m g \%)\end{array}$} & \multirow{2}{*}{$\begin{array}{l}\text { Platelet Count } \\
\text { per cmm }\end{array}$} & \multicolumn{2}{|c|}{ Patients } & \multicolumn{2}{|c|}{ Control } & \multicolumn{7}{|c|}{ Clotting Factor Assays } \\
\hline & & & $P T$ & $P T T$ & $P T$ & $P T T$ & II & $V$ & $V I I I$ & $I X$ & $X$ & $X I$ & $X I I$ \\
\hline 1 & 238 & 16,500 & $19 \cdot 0$ & $60 \cdot 4$ & $13 \cdot 2$ & 38.4 & & & & & & & \\
\hline 2 & 228 & 31,000 & $16 \cdot 2$ & $72 \cdot 8$ & $12 \cdot 5$ & $37 \cdot 6$ & 46 & 59 & 100 & 57 & 54 & & \\
\hline 3 & 347 & 111,000 & 15.6 & $49 \cdot 4$ & $12 \cdot 5$ & 41.6 & & & & & & & \\
\hline 4 & 359 & 102,000 & 13.0 & $215 \cdot 8$ & $13 \cdot 2$ & $42 \cdot 5$ & 45 & 66 & 48 & 2 & 17 & & 95 \\
\hline 5 & 542 & 265,000 & $14 \cdot 5$ & $120 \cdot 5$ & 13.0 & 55.0 & & & & & & & \\
\hline 6 & 318 & 24,000 & 15.8 & 255.0 & $12 \cdot 3$ & 43.0 & 50 & 32 & 10 & 37 & & & 77 \\
\hline 7 & 400 & 99,500 & $15 \cdot 8$ & $75 \cdot 0$ & 13.4 & $47 \cdot 4$ & 100 & 86 & 59 & 56 & & & 48 \\
\hline 8 & 235 & 97,500 & $17 \cdot 9$ & $71 \cdot 6$ & 13.9 & $44 \cdot 3$ & & & & & & & \\
\hline 9 & 280 & 16,500 & $19 \cdot 3$ & 61.9 & $14 \cdot 5$ & $48 \cdot 2$ & & & & & & & \\
\hline 10 & 290 & 154,000 & $120 \cdot 0$ & 180.0 & 14.9 & $42 \cdot 0$ & & & & & & & \\
\hline 11 & 650 & 101,500 & $25 \cdot 4$ & 93.4 & $13 \cdot 5$ & $48 \cdot 3$ & 100 & 63 & 35 & & & 26 & 15 \\
\hline 12 & 320 & 8,000 & $22 \cdot 0$ & $86 \cdot 2$ & $12 \cdot 5$ & $41 \cdot 0$ & & & & & & & \\
\hline 13 & 355 & 8,000 & $19 \cdot 0$ & $72 \cdot 6$ & 13.0 & $44 \cdot 3$ & 45 & 35 & 67 & & & 23 & 90 \\
\hline 14 & 160 & 29,000 & $16 \cdot 2$ & $132 \cdot 0$ & 13.6 & $41 \cdot 6$ & & & & & & & \\
\hline 15 & 165 & 3,000 & $37 \cdot 0$ & 263.4 & $13 \cdot 3$ & $46 \cdot 2$ & 23 & 5 & 3 & & & 6 & 18 \\
\hline 16 & 150 & 57,000 & $17 \cdot 8$ & $277 \cdot 0$ & $14 \cdot 3$ & 55.9 & & & & & & & \\
\hline 17 & 355 & 116,000 & $15 \cdot 2$ & $49 \cdot 0$ & $13 \cdot 7$ & $44 \cdot 7$ & & & & & & & \\
\hline 18 & 184 & 13,000 & $26 \cdot 4$ & $207 \cdot 0$ & $13 \cdot 3$ & 44.9 & 6 & 12 & 54 & & & 23 & 29 \\
\hline 19 & 355 & 110,000 & $15 \cdot 2$ & $49 \cdot 0$ & $14 \cdot 5$ & $44 \cdot 8$ & & & & & & & \\
\hline 20 & 184 & 12,500 & $26 \cdot 4$ & $207 \cdot 0$ & $13 \cdot 5$ & $46 \cdot 8$ & 6 & 12 & 54 & & & 23 & 29 \\
\hline 21 & 50 & 18,000 & 33.0 & $240 \cdot 0$ & $14 \cdot 1$ & $40 \cdot 5$ & & & & & & & \\
\hline 22 & 605 & 83,000 & 14.9 & $50 \cdot 5$ & $14 \cdot 3$ & $46 \cdot 5$ & & & & & & & \\
\hline 23 & 367 & 3,500 & 16.0 & 116.9 & $13 \cdot 1$ & $48 \cdot 2$ & & & & & & & \\
\hline 24 & 240 & 15,500 & $52 \cdot 5$ & $107 \cdot 4$ & 12.9 & $35 \cdot 7$ & & & & & & & \\
\hline 25 & 525 & 29,500 & $16 \cdot 6$ & $76 \cdot 6$ & $14 \cdot 6$ & $48 \cdot 5$ & & & & & & & \\
\hline 26 & 325 & 140,000 & $14 \cdot 4$ & $44 \cdot 9$ & $14 \cdot 2$ & $54 \cdot 7$ & & & & & & & \\
\hline 27 & 465 & 31,000 & $2 \cdot 22$ & $86 \cdot 6$ & $12 \cdot 8$ & $47 \cdot 1$ & & & & & & & \\
\hline 28 & 436 & 33,500 & $17 \cdot 4$ & 52.0 & $12 \cdot 7$ & $47 \cdot 3$ & & & & & & & \\
\hline 29 & 310 & 12,000 & $30 \cdot 6$ & $94 \cdot 2$ & $14 \cdot 5$ & $55 \cdot 5$ & & & & & & & \\
\hline Average & 305 & 59,000 & $24 \cdot 5$ & $119 \cdot 0$ & $13 \cdot 5$ & 43.9 & 46 & 41 & 48 & 38 & 35 & 20 & 50 \\
\hline
\end{tabular}

Table I Clotting factor depletions noted in patients treated in the Walter Reed Hospital shock unit

PT, prothrombin time; PTT, partial thromboplastin time, both in seconds. Factor assays are percentages of normal. 


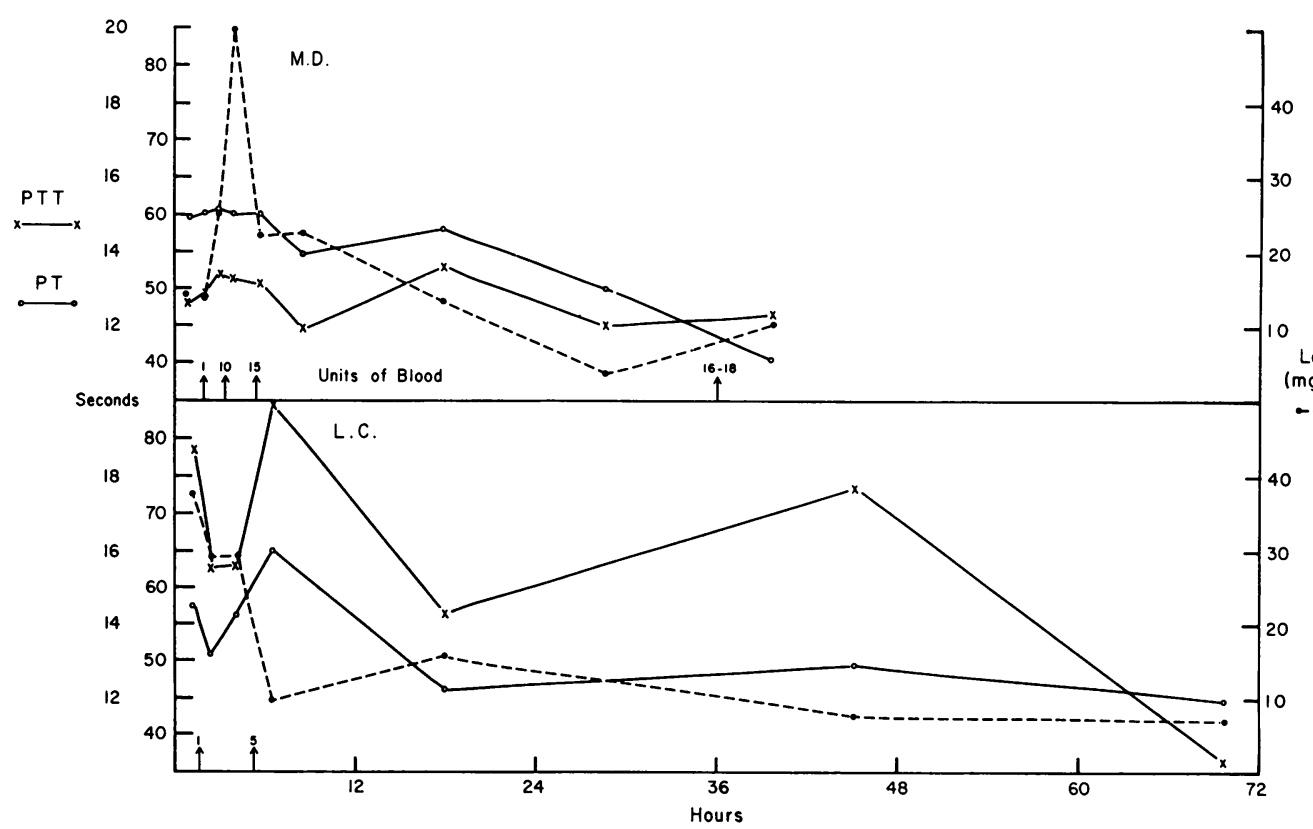

Fig. 13 Serial blood clotting and lactate studies in two casualties. Patient M.D. retained clotting stability despite rapid transfusion of 15 units of blood in three hours. He was never significantly hypotensive. Patient L.C. entered the hospital hypotensive with abnormal clotting studies before transfusion. He illustrates a type of response to transfusion that might be seen in factor-deficient individuals.

PT, prothrombin time; PTT, partial thromboplastin time.

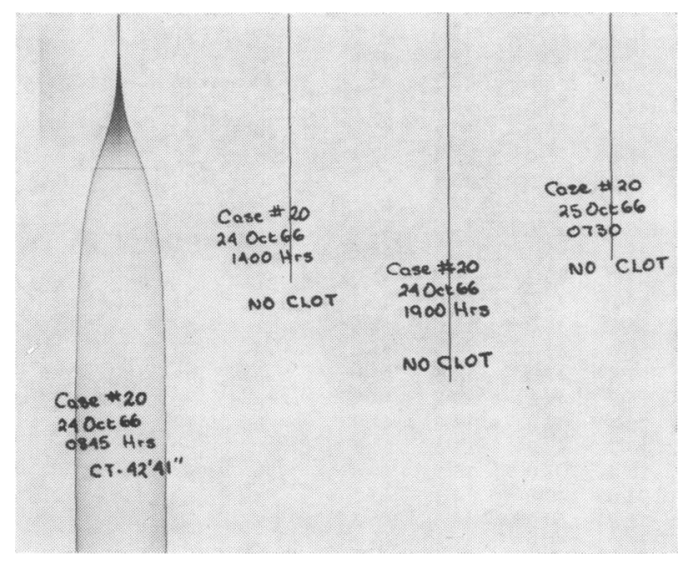

Fig. 14 Thromboelastogram on a patient studied in the shock unit. On admission the blood clotted although the clotting time was somewhat prolonged and the clot not as firm as normal. However, the patient deteriorated and the next three thromboelastograms failed to show any clotting at all. The patient died the following day.

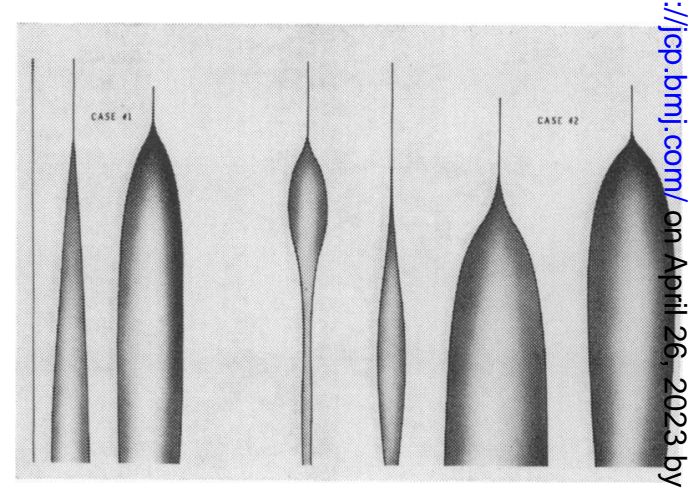

Fig. 15 On the left are three thromboelastograms from a patient treated on the shock ward. On admission (far left) his blood failed to coagulate in the thromboelastogram; this improved to normal on successive days and the patient recovered. On the right is a group of four thromboelastograms on another patient. On admission (left) the blood failed to clot normally and the poor clot lysed due to endogenous fibrinolysin as denoted by the narrowing 0 of the figure below. On subsequent days the thromboelastogram reverted to normal and the patie recovered. 
Vietnam, 27 of whom showed some clotting defect. All required transfusion but the multiple transfusions could not account for the clotting defect.

The plasma fibrinogen levels did not vary greatly during the acute phase of injury and transfusion and formed no recognizable pattern or correlations except for a steady rise beginning one or two days following injury.

These patients had at least four different factors influencing the clotting mechanism: 'shock', multiple transfusions of stored citrated blood, extensive local tissue damage, and tissue (and possibly thromboplastin) embolization. Each factor appears to have a positive correlation with the clotting defect (Table II).

Significant hypotension (systolic pressure by cuff measurement less than $90 \mathrm{~mm} \mathrm{Hg}$ for more than 20 minutes) was associated with a clotting defect though hypotension during anaesthesia did not seem to have the same significance (vasodilatation) (Tables II and III). The rise in lactate during an hypotensive episode under anaesthesia was often slight, indicating better tissue perfusion than during similar hypotension in an unanaesthetized patient.

Among 34 patients receiving up to 15 units of blood, the clotting studies were normal in 18 and abnormal in 16 cases (Tables IV and V). In contrast, each of the 11 patients receiving more than 15 units of blood had clotting abnormalities in the period after injury. Unfortunately, other major variables cannot be separated, because all 11 had also been hypotensive before anaesthesia and none had normal postoperative blood oxygen tensions.

Twenty-one of the 27 patients with clotting defects suffered blast or high velocity missile injuries with major long-bone fractures. One of the remaining six sustained a severe closed abdominal injury and cardiac arrest during operation. Among the 18 patients with no clotting defects, 14 sustained blast or high velocity missile injury, but only six had major long-bone fractures, possibly indicating less extensive local damage in the group with no clotting defects.

There was more definite evidence for an association between embolization and a clotting defect (Table VI). Excluding patients with chest and abdominal injuries, seven of 19 patients with clotting defects had unexplained preoperative arterial hypoxaemia, and therefore presumed embolization. Only three of 12 patients without clotting defect had evidence of embolization. If postoperative hypoxaemia is also considered, and this has many more variables, only three of the 19 subjects with clotting defects retained normal blood oxygen tensions throughout, compared with seven of the 12 without clotting defect.

Most of the patients studied had a combination of the four factors mentioned. Extensive tissue damage was common in both groups, but the other three factors were less common. Each of the

\begin{tabular}{lcc}
\hline & No Defects & $\begin{array}{l}\text { Clotting } \\
\text { Defect }\end{array}$ \\
\hline Total cases & 18 & 27 \\
'Shock' & 3 & 3 \\
Transfusion > 10 units & 1 & 0 \\
Presumed embolization & 2 & 1 \\
Shock + transfusion & 0 & 10 \\
Shock + embolization & 0 & 3 \\
Embolization + transfusion & 1 & 1 \\
Shock + embolization + transfusion & 0 & 2 \\
None of the above & 11 & 7 \\
\hline
\end{tabular}

Table II Presence or absence of clotting defects in 45 patients related to shock, transfusion of more than 10 units, and presumed embolization or various combinations

\begin{tabular}{lrl}
\hline & No Defects & $\begin{array}{l}\text { Clotting } \\
\text { Defect }\end{array}$ \\
\hline Total cases & 18 & 27 \\
Hypotensive before anaesthesia & 3 & 20 \\
Hypotensive only during anaesthesia & 4 & 2 \\
Never hypotensive & 11 & 5
\end{tabular}

Table III Clotting defects in 45 patients related to the occurrence of hypotension

\begin{tabular}{lll}
\hline Blood Transfusion (Units Blood) & No Defect & Clotting Defect \\
\hline $0-15$ & 18 & 16 \\
$15+$ & 0 & $11^{1}$
\end{tabular}

Table IV Defects in 45 patients related to extensive transfusion

${ }^{1}$ Also hypotensive and hypoxaemic

\begin{tabular}{lll}
\hline Blood Transfusion (Units Blood) & No Defect & Clotting Defect \\
\hline $1-9$ & 14 & 13 \\
$10-19$ & 4 & 5 \\
$20-30$ & 0 & 4 \\
$30+$ & 0 & 5
\end{tabular}

Table V Presence or absence of a blood-clotting defect in 45 patients related to the volumes of blood transfused

\begin{tabular}{lcc}
\hline Arterial Hypoxaemia & No Defect & Clotting Defect \\
\hline Total cases & 12 & 19 \\
Preoperative & 3 & 7 \\
Postoperative only & 2 & 8 \\
No hypoxaemia & 7 & 4
\end{tabular}

Table VI Patients with and without clotting defects according to presumed embolization (preoperative hypoxaemia) ${ }^{1}$

${ }^{1}$ Excluding patients with chest or abdominal injury.

three factors, 'shock', extensive transfusion (more than 10 units of blood in the first 24 hours), and embolization (unexplained preoperative hypoxaemia), was rare in the absence of the other two and no apparent association with a clotting defect was seen in these few patients (Table II). All 
combinations which included 'shock', however, were associated with a clotting defect.

No pattern of change associated with rapid transfusion emerged in this series: six normotensive patients given 6 to 15 units of blood rapidly had normal clotting studies at all times.

Figure 13 illustrates a shortening of clotting times during rapid transfusion from initially prolonged values, with subsequent prolongation several hours after cessation of transfusion. This kind of response is seen during temporary replacement of depleted clotting factors by transfused blood or blood products. The pattern is not that expected if bank blood were responsible for the bleeding problems often attributed to its use. Shortening of clotting times during rapid transfusion with later prolongation was seen in eight patients.

The more expected change, a lengthening of clotting times with rapid transfusion, was seen in six patients, but the clotting times decreased as transfusion was continued.

Finally, no definite pattern of change in fibrinogen levels could be detected. Any changes were relatively small in the acute phase. Of course all the patients were transfused with blood with normal fibrinogen levels. Fibrinogen levels often rose to about $1,000 \mathrm{mg}$ per $100 \mathrm{ml}$ on the second or third day after injury, especially in those with high velocity missile injuries. At this time the prothrombin and partial thromboplastin times were usually below normal.

In spite of the uncontrolled nature and multiplicity of the variables in this study, several conclusions seem warranted. Severely injured combat casualties often exhibit clotting abnormalities during the acute phase of injury. This appeared to be associated with extensive tissue damage, extensive transfusion of stored blood, presumed embolization at the time of injury, and haemorrhagic hypotension. Of all the factors, hypotension seems the most important. Combinations of factors, especially with prolonged hypotension, increased the likelihood of a clotting defect. Neither the aetiology nor the clinical significance of the clotting changes was apparent in this study except for an association with severely injured patients. Extremely abnormal clotting values were seen only in the few who died. Clinical bleeding problems were rare, but when they occurred it is not justifiable to attribute them to clotting aberrations due to replacement of the patient's blood with bank blood. Hypoperfusion appeared to be more important in our clinical study and also experimentally.

Most patients studied received stored bank blood for the treatment of oligaemia and during surgery. It is known to differ from normal circulating blood in many ways. In contrast to the situation in large civilian hospitals, the blood used in a field medical unit in a combat zone necessarily travels long distances, and usually undergoes several short periods with little or no refrigeration. Generator

failures, especially during the monsoon seaso $\frac{\widehat{\Omega}}{\text {; }}$ are expected occurrences and are accompanied by unavoidable variations in blood-bank-refrigeratơr temperatures. For these reasons it was of interest to estimate the clotting properties of the baik blood at its point of usage. Blood was drawn from the intravenous tubing during the latter half of transfusion and handled exactly as for arter samples except that additional citrate was n added for the clotting studies. All the blo@ studied was in Fenwal plastic bags said to contadi $450 \mathrm{ml}$ blood plus $67.5 \mathrm{ml} \mathrm{NIH}$ solution A. All the units of blood were administered through disposable field-type blood recipient sets (Fenwa or Burron).

The results are summarized in Table VII. Ba⿳亠口冋 blood, even old bank blood, has a relativeidy normal coagulation profile. In most cases it shoufd not contribute to a coagulation defect, but shouta actually help to correct it.

\begin{tabular}{llll} 
& $\begin{array}{l}\text { Partial Thromboplastin } \\
\text { Time } \\
(\mathrm{sec})\end{array}$ & $\begin{array}{l}\text { Prothrombin } \\
\text { Time } \\
(\mathrm{sec})\end{array}$ & $\begin{array}{l}\text { Fibrinogen } \\
(\mathrm{mg} \%)\end{array}$ \\
\hline $\begin{array}{l}\text { Old blood } \\
\text { Mean } \\
\text { Range }\end{array}$ & 65 & 18 & 390 \\
Fresh blood & 47 & $14-23$ & $290-5100$ \\
\hline
\end{tabular}

Table VII Measurements of bank blood administer ${ }_{d}$ at the 93rd Evacuation Hospital

'Old blood' represents 15 units of blood within five days off expiration, 8 units actually on the day of expiration. 'Fresh blo\& represents a single unit of blood given four hours after sheddio All blood used was supplied through the 406th Medical Laboratcy (Japan) and its mobile element in Vietnam.

On the basis of our studies, one conclusion cain be surely drawn: definite changes in the blogid clotting values of these young, acutely injured adults who require blood replacement, cannot attributed simply to the use of bank blood.

\section{Summary}

Severe or refractory shock due to traumfe, infection, or other cause is usually associated with an episode of disseminated intravascular coag柋 lation as manifested by the rapid development of a consumptive coagulopathy, and the finding of multiple intravascular thrombi associated with areas of focal necrosis in various vital organs.

References

Borgström, S., Gelm, L. E., and Zederfeldt, B. (1959). The formation of vena thrombi following tissue injury. An experimental study in rabbits. Acta chir. scand., Suppl., 247.

Bywaters, E. G. L. (1942). Crushing injury. Brit. med. J., 2, 6 616. Hardaway, R. M. (1968). Clinical Management of Shag
Thomas, Springfield, Ill. 